\title{
Les médecins aussi doivent de plus en plus développer leurs compétences de gestion
}

\author{
Le premier cours pilote pour la formation en gestion des médecins et des cadres \\ médicaux, organisé par la Société Suisse de Médecine Interne Générale en collabo- \\ ration avec la Fachhochschule Nordwestschweiz, s'est achevé en août 2013. Au total, \\ 21 participants ont suivi avec succès la formation continue «CAS - Management of \\ Medical Units». En raison de la grande demande, il est prévu de reconduire cette \\ offre de formation au printemps 2014.
}

\author{
Volker Schultea , Lukas Zemp ${ }^{b}$ \\ a Direction des cours, \\ Fachhochschule Nord- \\ westschweiz (FHNW) \\ b Secrétaire général de la SSMI
}

Le premier «CAS Management of Medical Units» 2013 a fait l'objet d'une évaluation continue par la direction du cours, qui est la Fachhochschule Nordwestschweiz (FHNW), pour le compte de la Société Suisse de Médecine Interne Générale (SSMI). Chaque contenu de cours du CAS a été évalué de manière critique par les participants. Le bilan du cours a été pleinement positif: ci-après, une rétrospective sur l'offre de cours CAS jusqu'ici existante, ainsi que sur quelques opinions des participants sur cette offre, formulées dans le cadre de l'évaluation qui a été faite du cours.

Thèmes centraux abordés dans les cours $\mathbf{2 0 1 3}$
Ce sont avant tout les thèmes du management inté-
gral, de la gestion du personnel, de la planification fi-
nancière et de la gestion comptable qui ont suscité le
plus vif intérêt. Dans le cadre de la thématique sur la
gestion du personnel, des réponses ont été apportées
aux questions suivantes: comment gérer les conflits
avec les collaborateurs, sous quelles conditions un li-
cenciement est-il possible et comment conduire un
entretien avec un collaborateur. Dans le cadre du mo-
dule sur la planification financière, des questions spé-
cifiques à la branche ont pu être posées, telles que le
conseil sur le choix de la forme juridique du cabinet
médical. Il est par exemple nécessaire de clarifier si une
société anonyme ou une Sarl constitue la forme juri-
dique la plus adéquate pour un cabinet médical. Les
bases du marketing ont été présentées dans le cadre du
module marketing. Ces bases couvrent l'ensemble des

Correspondance:

Lukas Zemp / SSMI

Solothurnerstrasse 68

CH-4008 Bâle

Tél. 0612259331

info[at]sgim.ch aspects qui sont importants dans la pratique quotidienne, comme par ex. une orientation client lorsque l'on s'occupe des patients, comment développer cette orientation client et comment parvenir à fidéliser sa clientèle. Cela englobe également des activités de communication marketing adaptées au groupe-cible pour les prestations médicales. Les bases du système de santé et de soins suisse et de la politique suisse en matière de santé ont été abordées au cours du module sur l'économie de la santé. Les participants ont eu un aperçu sur la situation actuelle des coûts, des prestations et des réformes du système de santé suisse. Ont également été traités l'évolution des primes, les tarifs, le principe EAE (efficacité, adéquation, économicité), ainsi que des thèmes tels que la gestion de l'offre, la rationalisation et le rationnement.

\section{Formation continue utile et transfert concret de la pratique}

Les médecins participants perçoivent le cours comme très axé sur la pratique. Christian $\mathrm{H}$., directeur d'établissements de soins, s'est exprimé en ce sens: «Le cours nous donne à nous médecins un premier aperçu, très intéressant, de la gestion d'entreprise». Wilhard K., directeur d'une centre de rééducation, souligne que: «Ce cours est extrêmement bien construit et est également compréhensible par les médecins. A l'avenir, je serai mieux en mesure de communiquer avec les personnes qui s'occupent de mes finances. C'est exactement ce que j'aurais toujours dû savoir lors de mes échanges avec mon directeur administratif.» Les participants jugent aussi important de recevoir le plus possible de recommandations concrètes et de checklists. Les différents commentaires des participants seront pris en compte pour la planification et la conception du deuxième cours CAS au printemps 2014.

Tous les participants au CAS ont rédigé un travail personnel. Près de la moitié des participants au cours avaient décidé de développer un plan d'affaire qu'ils pourraient appliquer à leur propre entreprise ou service. Les plans d'affaire ont été vérifiés par des professionnels et ont été présentés dans le cadre des cours. 